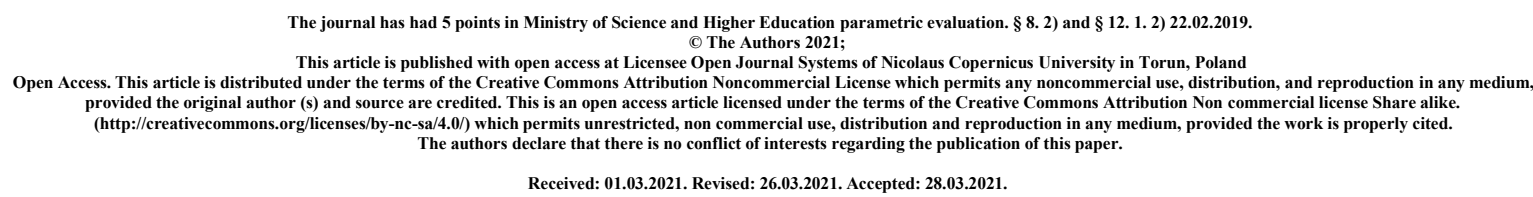

\title{
Effects of COVID-19 on doctors burnout
}

\section{${ }^{1}$ Edyta Medak}

Katedra i Zakład Psychologii

Uniwersytet Medyczny w Lublinie

20-081 Lublin

Poland

Principal contact for editorial correspondence.

\section{${ }^{2}$ Antoni Niedzielski}

Katedra i Zakład Psychologii

Uniwersytet Medyczny w Lublinie

20-081 Lublin

Poland

\section{Summary}

The impact of COVID-19 on the mental health of doctors who come into contact with suspects or sick people is being studied worldwide. Appropriate questionnaires sent by e-mail to the surveyed groups are used for this. The most common respondents are anaesthesiologists working at the ICU and HED. Women experience stress and anxiety more often than men. Physicians' mental health is affected by their age, workplace and access to personal protective equipment. A review of the literature shows the negative impact of the growing number of SARS-CoV-2 cases on the level of stress, anxiety and satisfaction with the work of doctors.

Key words: COVID-19, burnout, doctors, covid, fatigue, stress 


\section{INTRODUCTION}

Coronavirus-19 (COVID-19) is a highly contagious respiratory disease. The first case was registered on December 31, 2019 in Hubei Province, China. The rapid spread of the virus has turned it into a global health problem. In Poland, the first case was diagnosed on March 4, 2020 in Zielona Góra. Due to the drastically increasing number of cases, COVID-19 was declared a pandemic by the World Health Organization on March 11, 2020. [10, 13, 14] Faced with such a huge challenge as the time of the pandemic, doctors have become even more exposed to chronic mental stress than before. Undoubtedly, the high risk that doctors expose themselves to when diagnosing and treating infected patients, long, often exceeding normal working hours in a hospital, unpredictability of the virus, helplessness caused by lack of control, and finally death despite heroic struggle for life - these are some of the factors that can lead doctors to what is known as burnout. Its three main dimensions are: feeling of energy exhaustion or fatigue, increasing emotional distance from work or feeling of cynicism related to work, and reduced efficiency [17,19]. Exposure to this kind of symptoms can have a very negative impact on the ability and quality of services provided. health care system during the crisis caused by COVID-19 [7] Fear and living in a chronic threat to health and life affect the productivity of doctors and the level of care for patients [10].

\section{OBJECTIVE}

The aim of the study is to determine the impact of COVID-19 on doctors' burnout.

\section{REVIEW METHODS}

The publications available in the Scopus database were used for the literature review. A combination of English keywords was used: "burnout", "doctors", "covid", "fatigue", COVID19, stress. During the review of the materials, it was found that the subject of COVID-19 is widely used in English-language literature. The number of available articles from just six months reaches nearly 400. In turn, narrowing down the topic by combining it with professional burnout of doctors allows you to find more than 30 additional items. The work is a literature review of full-text publications only from 2020 . 


\section{REVIEW RESULTS}

COVID-19 has changed the psyche of doctors around the world. From January to April 2020, 240 specialists in the field of paediatrics, gynecology and surgery were tested in the city of Gujrat in the northern part of Pakistan. Of these, 98 people were female and 142 were male. All doctors worked with patients who were sick and diagnosed with the coronavirus. The study involved answering specific questions that allowed the assessment of changes in the mental health and behavior of doctors as a result of the outbreak of the global pandemic. The family members of each physician's friends were then asked about the physician's behavior change before and after the COVID-19 outbreak. The study found many negative changes in the mental health and behavior of doctors, including sleep problems, decreased appetite, irritability, decreased concentration, fatigue, loss of energy, and physical ailments such as abdominal pain. The risk factors causing the above changes include: prolonged on-call duty in crisis exacerbations, the need to maintain social distance, the use of protective equipment, fear of infecting family members, and prolonged discussions in social media regarding the current situation. It is worth noting that one doctor can be influenced by many risk factors [9].

The impact of the pandemic on the mental health of doctors was also tested in the United Kingdom. The questionnaire intended for this purpose was completed by a total of 207 gynecologists and obstetricians. There were significantly higher rates of depressive disorders and a higher level of generalized anxiety compared to the overall estimate. The analysis of additional variables showed that the level of anxiety was more common among women than among men. Respondents found that the most important factor influencing changes in workrelated mental health was the need to keep up with frequently changing COVID-19 guidelines and protocols. [16] The literature review shows that in Great Britain also anesthesiologists and those working in the first line, i.e. in HEDs and ICUs, are examined. The same examination and on doctors of the same specialty is also conducted in Ireland. The study was initiated to assess crisis response, the degree and frequency of mental stress and trauma during a pandemic. The influence of the mental state of the respondents on their professional performance is also examined. Two questionnaires are used for the study. The first is the 12item General Health Questionnaire (GHQ-12) that assesses the condition at a given moment and asks participants to compare it with their normal condition. In addition, it is characterized by high specificity and sensitivity, and its credibility has been confirmed in similar clinical trials on many populations. The second questionnaire is the Verified Event Impact Scale (IES$\mathrm{R}$ ), which is commonly used to measure post-traumatic stress disorder after a traumatic event. Both surveys are conducted via the internet platform. The study is long-term and has three phases: an accelerated study (at month zero), a peak study (7 days after the pandemic peak), and a delay study (30 days after the study peak). These three phases will allow the assessment of personal and professional factors affecting psychological well-being during the acceleration, peak, and slow-down phases of a pandemic. Due to the political differences regarding COVID-19 between the UK and Irish governments, differences are likely to occur in the pandemic wave. This can result in significant differences in the studied populations. Perhaps a joint analysis will not be possible. The decision will be made after the end of the study and detailed analysis of the results, and everything will be documented. Although the study is still underway, it is already certain that it will provide a reliable assessment of the 
psychological impact of the COVID-19 pandemic on frontline physicians, which will help with intervention and provide data for future emergency planning [14].

High rates of mental stress symptoms were confirmed in a cross-sectional study based on surveys of 193 anesthesiologists and ICUs from Cairo University hospitals. The study was conducted in the period from March 15 to April 15, 2020. More than half, as many as $65 \%$ of participants confirmed that the emergency situation related to the emergence of COVID-19 caused them to experience high levels of stress. The most common reasons were the fear of transmitting the disease to their relatives and the lack of a clear protocol for dealing with suspected or confirmed cases of the disease. [1] Anesthesiologists, due to the particularly high risk of contracting COVID-19 in emergency rooms, operating theaters and intensive care units, are probably one of the most studied and described groups of doctors in the literature. 512 Indian anesthetists actively involved in the care of coronavirus patients volunteered in a crosssectional study conducted online. Only 10 days were allowed to collect the data - from 12 to 22 May 2020. The questions included in the questionnaire concerned the likely causes of stress (anxiety and insomnia) during the pandemic. Anxiety was assessed on the generalized anxiety disorder scale (GAD-7), and insomnia according to the insomnia severity index (ISI). The results show that over $70 \%$ of respondents suffer from anxiety and over $60 \%$ suffer from insomnia. Based on the analysis of questionnaires, factors predisposing to the occurrence of these symptoms were: age $<35$ years old, female gender, people staying in residence, fear of infecting oneself or their family, fear of lower remuneration, fear of being alone during isolation, fear of losing accommodation and food problem. Insomnia was more common in unmarried doctors, and a higher level of anxiety was observed among married people. People who have been isolated from their loved ones and lonely are more likely to develop mental health problems. This confirms the importance of supporting family and friends and their positive impact on mental health. Due to the enormity of family-related tasks and often two jobs, women working as anesthesiologists had higher levels of stress and burnout than men. Prolonged isolation and quarantine lead to irritability, anxiety and, consequently, insomnia. Doctors, faced with the high challenges they face in caring for COVID-19 patients, are under chronic stress that impairs their ability to fall asleep and daytime sleepiness. This causes fatigue and results in less performance while working. [8] In early April 2020, when the number of COVID-19 cases in Iran was 32,612 and the demand for medical care was particularly high, a survey was launched to assess the mental health of medical staff and job satisfaction. The study lasted 15 days and identified risk factors leading to reduced job satisfaction and burnout during the outbreak. The Health Assessment Questionnaire (SF-12) shows that older workers are in better mental but not physical condition. Women feel more anxious and exhibit depressive behavior more often. Taking into account the workplace of the staff, it was proved that employees of private institutions were in a better mental state than those working in public institutions. Access to personal protective equipment has a positive effect on physical health, job satisfaction and the level of perceived stress. The feeling of insecurity has a destructive effect on mental health. Workers who were unsure if they had COVID-19 were found to be more stressed and anxious [21].

Currently, the largest world "leader" in terms of the number of cases of disease and deaths is the United States. With just $4.25 \%$ of the world's population, they account for as much as $30 \%$ of cases and $28 \%$ of deaths worldwide. How is it possible that such a result applies to the richest country in the world, with an average annual income of a doctor of $\$ 287,049.3$ ? The answer to this question is: burnout. The first national study on burnout was conducted there 
six years ago, when both the economy, medicine and society itself had significantly lower levels of stress than today. Even then, however, burnout was a problem. With over 7,000 of physicians who completed the Maslach inventory of burnout, $45.8 \%$ reported at least one symptom of burnout. Significant differences were observed in the degree of burnout depending on the specialization. The highest rates were recorded in family medicine, internal medicine and emergency medicine doctors. Physicians much more often than other working Americans showed symptoms of burnout and dissatisfaction with the work-life balance [15].

In January 2020, burnout was reported by $52.3 \%$ of American internists working in hospitals and $54.5 \%$ of outpatient internists. A significantly higher level of emotional exhaustion and depersonalization was found in the first group compared to the second. There were no differences in symptoms of depression in both groups. Doctors working in the hospital admitted that their work schedule leaves enough time for private life. It is amazing, according to ProPublica, that in the midst of the pandemic, American hospitals employ fewer doctors and reduce their salaries. In less than a month, COVID-19 made drastic cuts in hospital bills, increasing the amount of work and responsibility for those who stayed in them [15].

On February 8, 2020, in Singapore, one of the first countries outside China to report COVID19 cases, a so-called epidemic response inventory was introduced. Its goal was to limit contacts with other employees by creating teams with permanent members. In such a situation, if someone were infected, only the team would be quarantined. Teams were set up and assigned doctors according to their seniority and experience. A 12-hour operating mode has been introduced. Due to the concern for the mental health of Chinese medical personnel, mandatory breaks from work, a rest area and a 24-hour telephone peer support line were planned to provide care and help with problem solving. In order to assess the validity of the introduced changes, a survey was conducted in the seventh week, which showed approval of the work in the 12-hour system. The reasons are: greater safety due to less interaction between the teams and minimized consumption of PPE. The vast majority of respondents also appreciated more time to rest, better sleep quality and the opportunity to play sports, which is very important in the psychophysical aspect of coping with a pandemic [6].

In the period from February 19 to April 17, 2020, doctors from 5 major hospitals treating patients with COVID-19 in Singapore and India were examined. Using the anxiety and stress depression scale and the tools of the revised event impact scale, it was proved that $5 \%$ of respondents had moderate to very severe depression, $8 \%$ moderate to very severe anxiety, and almost $4 \%$ moderate to severe psychological stress. The most frequently reported symptom was headache and it concerned over $30 \%$ of the respondents [5]. The first case of COVID-19 in India occurred on January 30, 2020 in Kerala state. A cross-sectional observational study was conducted in hospitals designated for the care of patients. The problem of stigmatization among doctors was examined using online questionnaires. Over $62 \%$ of respondents achieved a higher level of perceived stigma, and $63 \%$ a higher level of perceived stress. It found that frontline doctors during the pandemic view themselves as stigmatized and feel rejected by others because of their contact with COVID-19 patients. Mental poor state as a result of the perceived stress leads to distraction when caring for patients [3.20].

A cross-sectional observational study was also conducted in West Bengal. It started on March 28, 2020 and ended on April 6, 2020. The questionnaires were sent by e-mail. The Depression, Anxiety and Stress Scales were used to assess the mental health of the doctors. 152 people 
participated in the study, $35 \%$ of whom were depressed, $40 \%$ experienced anxiety, and $33 \%$ reported stress. Most of the study participants had on-call duty lasting 6-12 hours, which is a positive finding. However, it should be remembered that with the passage of time and the spread of the pandemic, more and more doctors will be in quarantine, which will result in longer periods of working doctors' duty, and this may have a negative impact on their mental health. [4] COVID-19 poses an unprecedented threat to the physical and mental health of doctors. The constantly growing number of confirmed and suspicious cases, overwhelming workload, insufficient supply of personal protective equipment, publicizing the critical situation by the media, lack of specific medications, insufficient support, stigmatization and isolation have a destructive effect on the psyche of doctors. Increasing levels of stress and anxiety, along with the developing pandemic, lead to falling job satisfaction, and this can lead to what is known as burnout. However, collecting data on the negative impact of a pandemic on doctors does not help them survive this critical time. Rather, they need guidance to achieve mental well-being. Adequate leadership is essential and plays a key role in responding to COVID-19. Clearly defined actions to combat the pandemic will reduce stress and burnout among doctors $[2,11,18]$.

To reduce the risk of burnout among doctors, their stress level should be minimized. Providing high-quality protective materials during a pandemic significantly alleviates the employee's concerns about his own life and the health of his relatives. In Germany, the Federal Institute for Occupational Safety and Health recommends that all non-healthcare professionals wear only non-medical mouth and nose protection. Medical protection of the mouth and nose, together with certificates, should be reserved to healthcare professionals to prevent them from getting infected and possibly infecting other patients. Another reason is the fear of losing health workers due to insufficient protection [12]. In Malaysia, doctors were not adequately supplied with PPE at the start of the pandemic, resulting in a large number of infected doctors. The subsequent use of personal protective equipment shows that appropriate medical protection can effectively protect against SARS-CoV-2 and reduce the stress caused by the already difficult professional situation of doctors [12].

\section{LITERATURE}

1. Ali, H., Ismail, A.A., Abdalwahab, A.: Mental stress in anesthesia and intensive care physicians during COVID-19 outbreak. Anesthesiology and Pain Medicine. 2020; 10(5),e106623: 1-6.

2. Bäker, A., Bech, M., Geerts, J.: Motivating doctors into leadership and management: A cross-sectional survey. BMJ Leader.2020.

3. Bhattacharya, K., Bhattacharya, N.: Health care workers facing social ostracism during COVID-19. Journal of the Indian Medical Association . 2020; 118(8): 22-24.

4. Chatterjee, S.S., Bhattacharyya, R., Bhattacharyya, S.: Attitude, practice, behavior, and mental health impact of COVID-19 on doctors. Indian Journal of Psychiatry. 2020; 62(3): 257-265.

5. Chew, N.W.S., Lee, G.K.H., Tan, B.Y.Q.: A multinational, multicentre study on the psychological outcomes and associated physical symptoms amongst healthcare workers during COVID-19 outbreak. Brain, Behavior, and Immunity. 2020; 88: 559-565.

6. Chua, W.L.T., Quah, L.J.J., Shen, Y.: Emergency department $€$ outbreak rostering\&\#39; to meet

challenges of COVID-19. Emergency Medicine Journal. 2020; 37(7): 407-410.

7. DE LUCA, R., Calabrò, R.S.: How the COVID-19 pandemic is changing mental health disease management: The growing need of telecounseling in Italy. Innovations in Clinical 
Neuroscience. 2020; 17(4-6):16-17.

8. Jain, A., Singariya, G., Kamal, M.: COVID-19 pandemic: Psychological impact on anaesthesiologists. Indian Journal of Anaesthesia. 2020; 64(9): 774-783.

9. Mahmood, S., Choudhary, A.N., Bharo, M.A.: Psychological Changes in Doctors Working in Pediatrics, Gynaecology, Medicine and Surgery during Corona Pandemic (Covid-19).

Pakistan Journal of Medical and Health Sciences. 2020; 14(2): 368-370.

10. Majeed, A., Molokhia, M., Pankhania, B., Asanati, K.: Protecting the health of doctors during the COVID-19 pandemic. British Journal of General Practice. 2020; 70(695): 268-269. 11. Neto, M.L.R., Almeida, H.G., Esmeraldo, J.D.: When health professionals look death in the eye: the mental health of professionals who deal daily with the 2019 coronavirus outbreak. Psychiatry Research . 2020; 288,112972.

12. Nienhaus, A., Hod, R.: COVID-19 among health workers in germany and Malaysia. International Journal of Environmental Research and Public Health. 2020; 17(13),4881: 1-10. 13. Nowicki, G.J., Ślusarska B., Tucholska K.: The severity of traumatic stress associated with covid-19 pandemic, perception of support, sense of security, and sense of meaning in life among nurses: Research protocol and preliminary results from poland. International Journal of Environmental Research and Public Health.2020; 17(18),6491: 1-18.

14. Roberts, T., Daniels, J., Hulme, W.: COVID-19 emergency response assessment study: A prospective longitudinal survey of frontline doctors in the UK and Ireland: study protocol. BMJ Open. 2020; 10(8),039851.

15. Schwartz, S.A.: Covid-19 and the documented failure of the American illness profit system - We have to stop treating our doctors, nurses, healthcare workers, and ourselves this way. Explore. 2020; 16(4): 210-213.

16. Shah, N., Raheem, A., Sideris, M., Velauthar, L., Saeed, F.: Mental health amongst obstetrics and gynaecology doctors during the COVID-19 pandemic: Results of a UK-wide study. European Journal of Obstetrics and Gynecology and Reproductive Biology. 2020; 253: 90-94.

17. Shaw, S.C.K.: Hopelessness, helplessness and resilience: The importance of safeguarding our trainees\&\#39; mental wellbeing during the COVID-19 pandemic. Nurse Education in Practice.

2020; 44,102780.

18. Simons, G., Baldwin, D.S.: Covid-19: Doctors must take control of their wellbeing. The BMJ. 2020; 369,m1211.

19. Szemik, S., Gajda, M., Kowalska, M.: The review of prospective studies on mental health and the quality of life of physicians and medical students | [Przegląd badań prospektywnych na temat stanu zdrowia psychicznego oraz jakości życia lekarzy i studentów medycyny]. Medycyna Pracy. 2020; 71(4): 483-491.

20. Uvais, N.A., Shihabudheen, P., Hafi, N.A.B.: Perceived Stress and Stigma Among Doctors Working in COVID-19-Designated Hospitals in India. The primary care companion for CNS disorders. 2020; 22(4).

21. Zhang, S.X., Liu, J., Afshar Jahanshahi, A.: At the height of the storm: Healthcare staff\&\#39;s

health conditions and job satisfaction and their associated predictors during the epidemic peak of COVID-19. Brain, Behavior, and Immunity. 2020; 87: 144-146. 